\title{
Hydrostatic Balloon Dilation of Congenital Esophageal Stenoses Associated With Esophageal Atresia
}

\author{
By Nicholas A. Shorter, David P. Mooney, Therese J. Vaccaro, and Steven K. Sargent \\ Lebanon, New Hampshire
}

It has been stated that congenital cartilage rings in the esophagus do not respond to dilation and should be resected. The authors report on 3 infants with congenital esophageal stenoses who were treated successfully with hydrostatic balloon dilation. Based on the appearance during dilation the authors believe that these stenoses were carti- lage rings. The technique is described in detail. Balloon dilation is the treatment of choice for these patients. Resection should be reserved for those who do not respond to this form of therapy.

$J$ Pediatr Surg 35:1742-1745. Copyright (0) 2000 by W.B. Saunders Company.
$A^{\prime}$ LTHOUGH RARE, congenital esophageal stenosis (CES) is being recognized more frequently in association with esophageal atresia. Whether this is because of greater awareness or reflects a true increased incidence of this abnormality is not clear. Three subtypes have been described ${ }^{1}$ : (1) ectopic tracheobronchial tissue, including cartilage, in the wall of the esophagus; (2) a membranous diaphragm; and (3) segmental hypertrophy of the muscularis and submucosal layers with fibrosis (fibromuscular stenosis). Current consensus opinion is that the last of these may respond to dilation but the other two require resection. ${ }^{1-3}$ Although it is true that the majority of resected specimens have shown tracheobronchial remnants, the fact remains that if dilation is successful, no specimen is available, so a definitive subtype cannot be determined in those cases, and although endoscopy may be able to diagnose a web, differentiation of a CES caused by ectopic cartilage from one caused by fibromuscular hypertrophy is not possible from either the endoscopic or conventional radiologic appearances. Thus, the percentage of each type that will respond to dilation is not actually known. In addition, most dilation attempts in the past have used bougienage, which applies a shearing force to the stricture in addition to a radial one and is, therefore, less efficient and more likely to produce damage to the esophageal wall.

We present 3 infants with CES who were treated

From the Departments of Surgery, Pediatrics, and Radiology, Children's Hospital at Dartmouth, Lebanon, $\mathrm{NH}$.

Address reprint requests to Nicholas A. Shorter, MD, Department of Surgery, Memorial Sloan-Kettering Cancer Center, 1275 York Ave, New York, NY 10021.

Copyright $@ 2000$ by W.B. Saunders Company

0022-3468/00/3512-0011\$03.00/0

doi:10.1053/jpsu.2000.19238 successfully with hydrostatic balloon dilation. Based on the appearance during dilation, the presence of a short, sharp, "waistlike" impression on the balloon, which suddenly disappeared as pressure was increased (Fig 1), we believe that these represent cartilage rings, and that

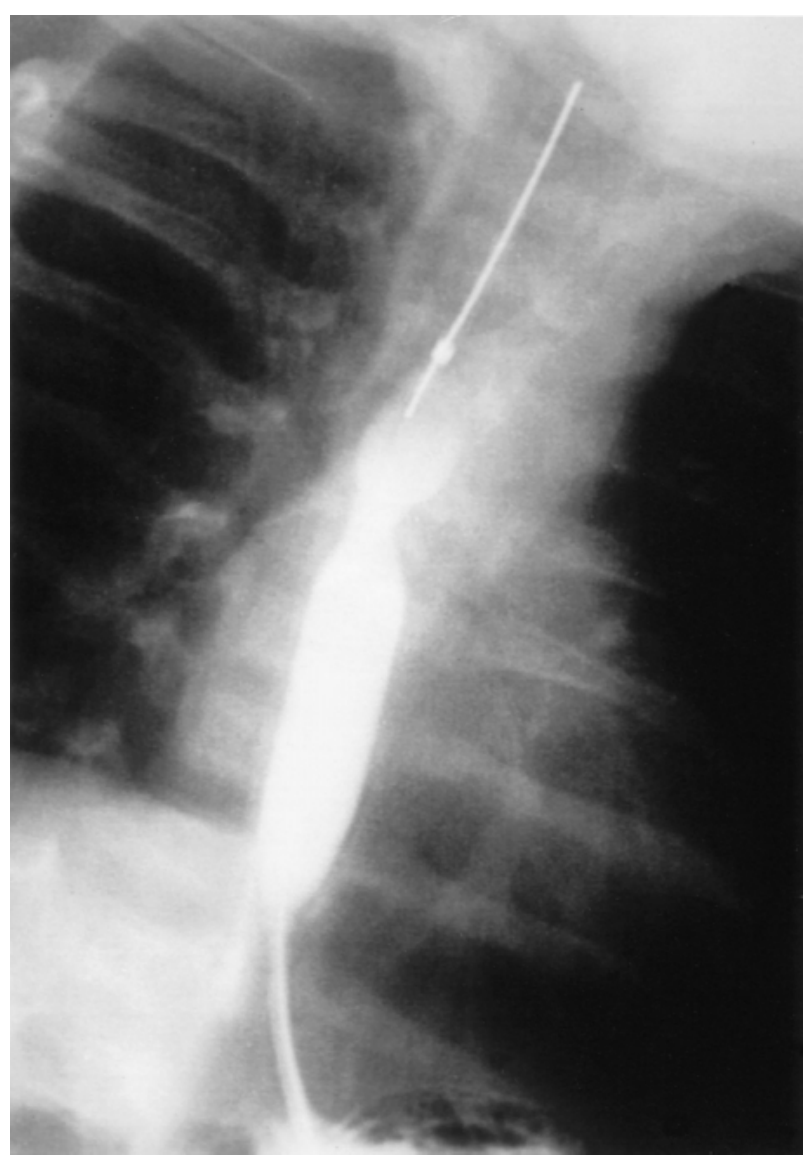

Fig 1. Case 3 shows the characteristic waistlike impression on the balloon. 


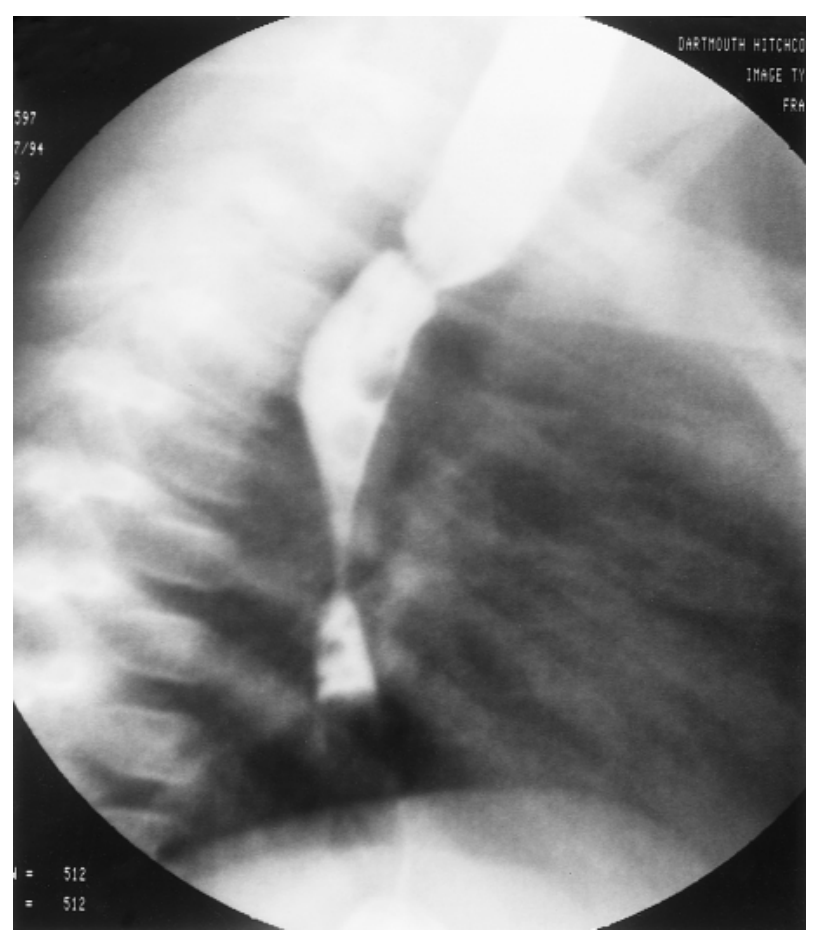

Fig 2. Esophagram of case 1 shows patent anastomosis and distal stenosis.

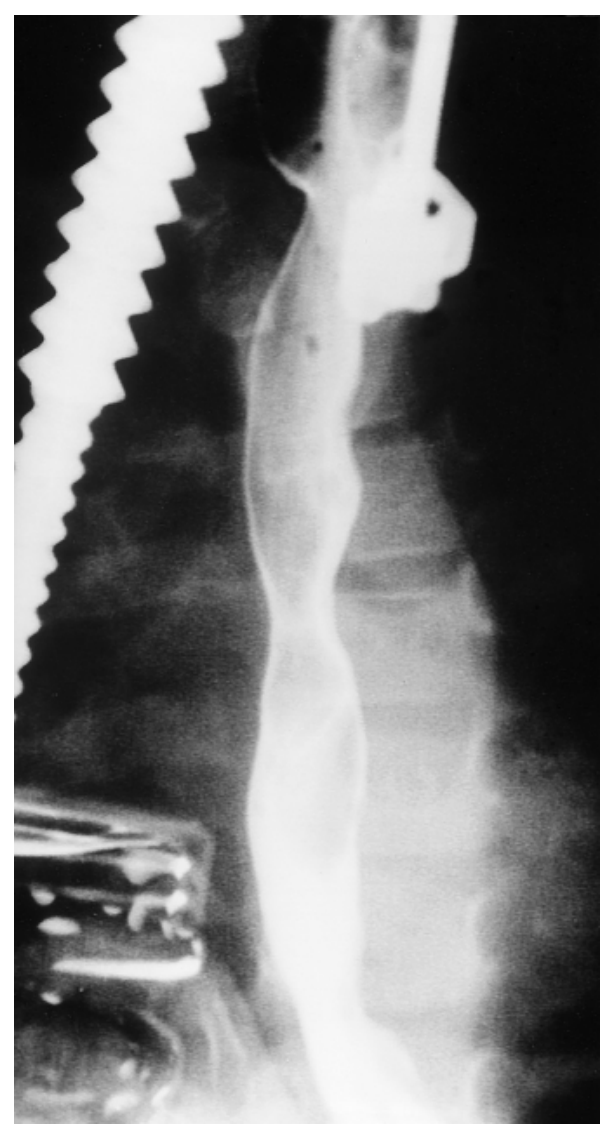

Fig 3. Follow-up esophagram of case 1, 4 years and 8 months after dilation. hydrostatic balloon dilation should be the initial treatment for all forms of CES.

\section{CASE REPORTS}

\section{Case 1}

A 36-week, 2,130-g girl was born with proximal esophageal atresia and a distal tracheoesophageal fistula. Primary repair was performed, and at that time an $8 \mathrm{~F}$ catheter would not pass down the distal esophageal segment. A 5F catheter passed easily. Using dilators, the stricture was sized to $2.5 \mathrm{~mm}$ in width and 1 to $2 \mathrm{~mm}$ in length. The mucosa was examined using a small cystoscope, and it was normal. It was possible to dilate the stricture to $3 \mathrm{~mm}$, at which point an $8 \mathrm{~F}$ catheter would pass through it. A primary anastomosis of the esophagus was then performed, and a gastrostomy was placed. Postoperatively, the infant did well, and a subsequent esophagram confirmed the presence of the distal stricture (Fig 2). Balloon dilation was performed at 14 weeks of age, and oral feedings were begun at that time without difficulty. Now, at 6 years old, she has had no further problems and has required no further dilations (Fig 3).

\section{Case 2}

A 39-week, 3,070-g boy underwent primary repair of proximal esophageal atresia and distal tracheoesophageal fistula. A prenatal diagnosis of possible esophageal atresia had been made by ultrasound

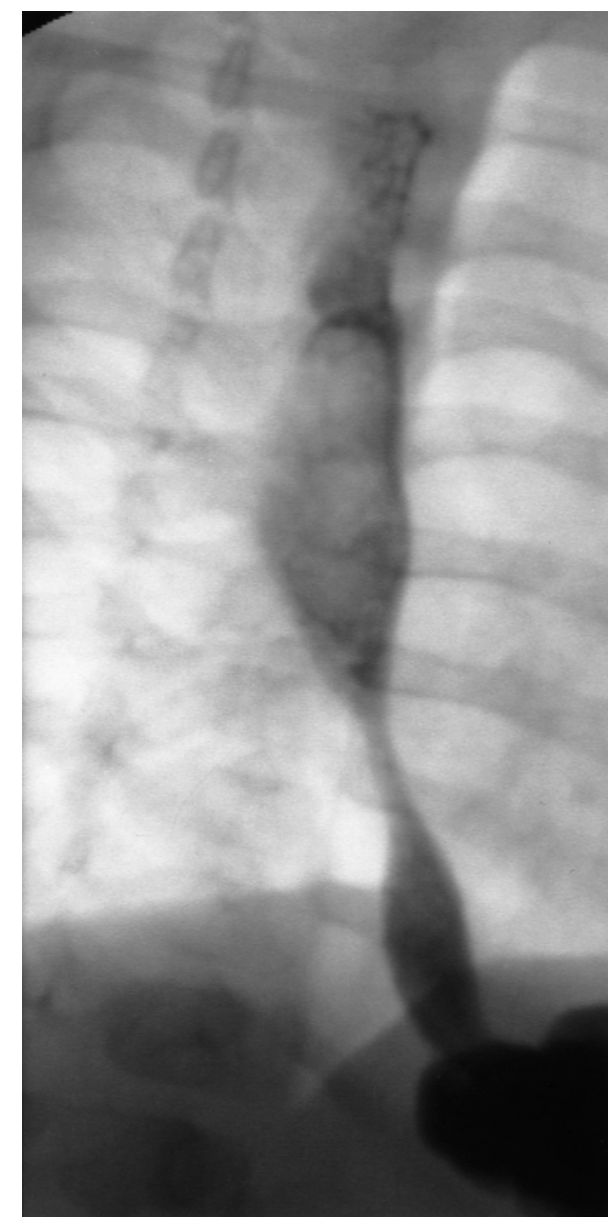

Fig 4. Esophagram of case 2 shows patent anastomosis and distal stenosis. 


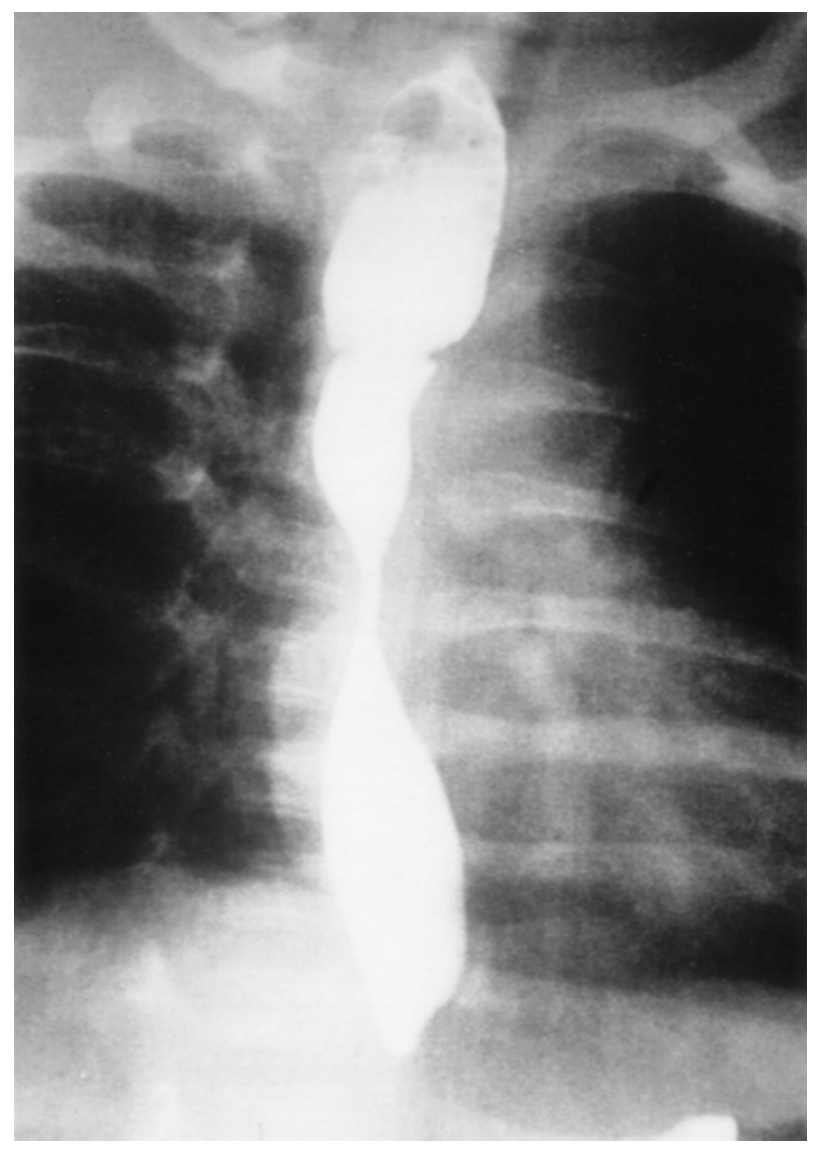

Fig 5. Esophagram of case 3 shows patent anastomosis and distal stenosis.

scan based on polyhydramnios and an absent stomach bubble. Intraoperatively, a $10 \mathrm{~F}$ catheter was passed successfully down the distal esophageal segment. He did well until 10 months of age when feeding difficulties developed, and a contrast study found a distal CES (Fig 4). There was no evidence of reflux. Balloon dilation was performed and repeated 4 weeks later. Now, at 4 years old, he has had no further difficulties.

\section{Case 3}

A 37-week, 3,230-g girl underwent primary repair of proximal esophageal atresia with distal tracheoesophageal fistula. As in case 1 , an $8 \mathrm{~F}$ catheter would only pass a short distance down the distal esophageal segment, whereas a $5 \mathrm{~F}$ catheter passed easily into the stomach. For that reason a gastrostomy was performed after completion of the primary repair. There were no postoperative complications, and an esophagram confirmed the presence of the distal CES (Fig 5) Balloon dilation was performed successfully at 7 weeks of age (Figs 1 and 6). Oral feedings were begun at that time without difficulty.

\section{TECHNIQUE}

The dilations were done in the diagnostic radiology suite without need of general anesthesia. Two patients had gastrostomies placed at the time of surgical repair, which greatly simplified the procedure, because it could be performed in a retrograde manner through the gastrostomy site.
At the time of stricture dilation, a preliminary contrast swallow was performed using a nonionic water-soluble agent, Omnipaque 240 (Iohexol), to localize the stricture and reassess its severity (diameter). A straight, floppy tipped, 0.035-inch standard angiographic guidewire was advanced under fluoroscopic guidance into the esophagus and through the stricture. A balloon dilation catheter (Boston Scientific Corporation, Watertown, MA) with a 3- to 4-cm long balloon and with at least a 10 ATM pressure limit was advanced over the guidewire until the balloon spanned the distal esophageal stricture. For the initial dilation, a balloon with an inflation diameter approximately $2 \mathrm{~mm}$ greater than the diameter of the stricture was chosen. The balloon was inflated gradually with dilute contrast over the guidewire, the tip of which remained in the esophagus to prevent the stiff tip of the balloon catheter from injuring the esophageal wall. If the balloon reached maximum diameter, and no waist had appeared on the balloon, the procedure was repeated with a 2-mm larger one. Duration of inflation was 1 minute.

In all 3 cases, the waist appeared and then very suddenly disappeared at or before an 8-mm-sized balloon. The maximum pressure needed to "fracture" the

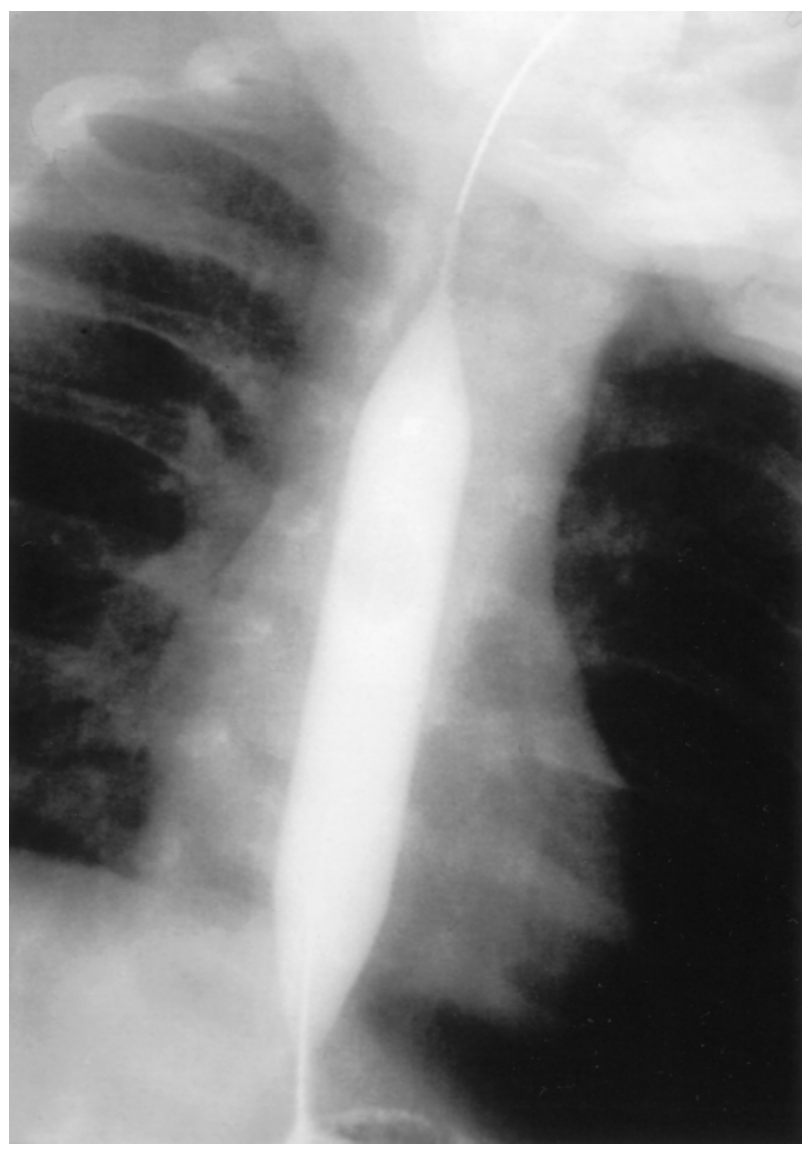

Fig 6. Case 3 shows $10-\mathrm{mm}$ balloon fully inflated. The waist is no longer visible. 
stricture was 10 ATM; 2 cases fractured at 3 to 4 ATM of pressure (These pressures are similar to those used by Goldthorn et $\mathrm{al}^{4}$ in successfully dilating esophageal anastomotic strictures but significantly higher than the 1 ATM used with only limited success by Neilson et $\mathrm{al}^{3}$.) The esophagus was then progressively dilated to a 10- or 12-mm diameter balloon, after which the last balloon was fully deflated, the guidewire removed, and nonionic water-soluble contrast (Omnipaque 240) injected through the catheter end hole as the catheter was withdrawn, to check for extravasation. The infant was admitted to the hospital overnight for observation, and oral feedings were begun the following day.

\section{DISCUSSION}

Congenital esophageal stenosis caused by ectopic tracheobronchial remnants was first reported by Frey and Duschel in $1936 .{ }^{2}$ The first report of CES associated with esophageal atresia was by Dunbar in $1958,{ }^{5}$ and up to 1994, less than 50 cases had been described, indicating that it is a rare abnormality. In contrast, Sheridan and Hyde $^{6}$ identified 4 cases in a series of 50 consecutive esophageal atresia patients, and we have seen 3 in a consecutive series of 13 , suggesting that it may be a more common association than previously thought.

All 3 of our patients responded well to balloon dilation, and, unlike previous reports of successful bougienage, multiple dilations were not necessary. The 1 patient who was dilated twice was dilated on the first occasion to $9 \mathrm{~mm}$ and then on the second to $10 \mathrm{~mm}$. There was no evidence of restenosis at the time of the second dilation, and, in retrospect, dilation to $10 \mathrm{~mm}$ could and should have been done on the first occasion. One can, of course, argue that these were not cartilage rings. However, we believe that the radiologic appearance during dilation (ie, the short, sharp, waistlike impression on the balloon, which suddenly disappears) indicates that they were, and this interpretation is endorsed by others. ${ }^{3}$ Although tracheobronchial remnants are most commonly located in the lower distal third of the esophagus, ${ }^{1}$ they also occur at the level seen in these 3 patients. $3,7,8$

Hydrostatic dilation of CES is safe, simple, and effective. Resection should be reserved for those who fail this form of therapy.

\section{REFERENCES}

1. Nihoul-Fékété C, De Backer A, Lortat-Jacob S, et al: Congenital esophageal stenosis: A review of 20 cases. Pediatr Surg Int 2:86-92, 1987

2. Yeung CK, Spitz L, Brereton RJ, et al: Congenital esophageal stenosis due to tracheobronchial remnants: A rare but important association with esophageal atresia. J Pediatr Surg 27:852-855, 1992

3. Neilson IR, Croitoru DP, Guttman FM, et al: Distal congenital esophageal stenosis associated with esophageal atresia. J Pediatr Surg 26:478-482, 1991

4. Goldthorn JF, Ball WS, Wilkinson LG, et al: Esophageal stric- tures in children: Treatment by serial balloon catheter dilatation. Radiology 153:655-658, 1984

5. Margarit J, Castanõn M, Ribó JM, et al: Congenital esophageal stenosis associated with tracheoesophageal fistula. Pediatr Surg Int 9:577-578, 1994

6. Sheridan J, Hyde I: Oesophageal stenosis distal to oesophageal atresia. Clin Radiol 42:274-276, 1990

7. Spitz L: Congenital esophageal stenosis distal to associated esophageal atresia. J Pediatr Surg 8:973-974, 1973

8. Murphy SG, Yazbeck S, Russo P: Isolated congenital esophageal stenosis. J Pediatr Surg 30:1238-1241, 1995 